\title{
Possible anticipation in familial epidermolytic palmoplantar keratoderma with the p.R163W mutation of Keratin 9
}

\author{
Y. Guo ${ }^{1}$, M. Shi ${ }^{2}$, Z.P. Tan ${ }^{3}$ and X.L. Shi ${ }^{1}$ \\ ${ }^{1}$ Department of Internal Medicine, \\ The Second Xiangya Hospital of Central South University, \\ Changsha, Hunan Province, China \\ ${ }^{2}$ Department of Ophthalmology, \\ The Second Xiangya Hospital of Central South University, \\ Changsha, Hunan Province, China \\ ${ }^{3}$ Clinical Center for Gene Diagnosis and Therapy of State Key Laboratory of \\ Medical Genetics, The Second Xiangya Hospital of Central South University, \\ Changsha, Hunan Province, China \\ Corresponding author: X.L. Shi \\ E-mail: drxiaoliushi@sina.cn
}

Genet. Mol. Res. 13 (4): 8089-8093 (2014)

Received July 23, 2013

Accepted November 28, 2013

Published October 7, 2014

DOI http://dx.doi.org/10.4238/2014.October.7.3

\begin{abstract}
Epidermolytic palmoplantar keratoderma (EPPK) is an autosomal dominant disease characterized by diffuse hyperkeratosis of the epidermis of the palm and sole with an erythematous margin. The Keratin 9 (KRT9) and Keratin 1 genes are responsible for EPPK. Several previous studies have focused on the genetic basis of EPPK; however, genetic anticipation has not yet been reported. We described a four-generation family with EPPK and identified a p.R163W mutation of KRT9. We observed a decrease in the age of onset in three consecutive generations in the family of the proband, indicating possible genetic
\end{abstract}


anticipation in this familial EPPK. Further studies are needed to elucidate the mechanisms of anticipation in EPPK.

Key words: Epidermolytic palmoplantar keratoderma; Keratin 9; Keratin 1; Anticipation

\section{INTRODUCTION}

Epidermolytic palmoplantar keratoderma (EPPK; MIM \#144200) is an autosomal dominant $(\mathrm{AD})$ disease characterized by diffuse hyperkeratosis of the epidermis of the palm and sole with an erythematous border (Reis et al., 1994). The genetic background of EPPK has been established. The Keratin 9 gene (KRT9) is the major disease-causing gene responsible for EPPK (Hennies et al., 1994; Reis et al., 1994). However, mutations of the Keratin 1 gene (KRTI) have also been reported in some atypical EPPK families (Terron-Kwiatkowski et al., 2004; Liu et al., 2009). In this study, we present genetic investigations of a family from Hunan Province in South-Central China.

\section{MATERIAL AND METHODS}

\section{Patients}

The pedigree is a four-generation family including 6 affected and 30 unaffected individuals (Figure 1A). The proband (IV-7 in pedigree), a three-year-old girl, presented at the Department of Cardiothoracic Surgery, The Second Xiangya Hospital of Central South University, Changsha, China for a heart murmur. She was diagnosed with an 8-mm peri-membranous ventricular septal defect (VSD) by two-dimensional color Doppler echocardiography. Subsequently, the congenital heart defect (VSD) was surgically corrected. None of the other members of the family have cardiac defects. At the first examination of the proband, hyperkeratosis on the palms and soles were observed (Figure 1B). Her mother also had hyperhidrosis of the palms and soles (III-9 in pedigree; Figure 1B). Biopsy results from the mother's left palm showed hyperkeratosis, and subsequently, the diagnosis of EPPK was confirmed by the Department of Pathology of the Second Xiangya Hospital.

\section{Genotyping}

Informed written consent for blood sample collection was obtained from all participants of the family. Genomic DNA was prepared using a DNeasy Blood \& Tissue Kit (Qiagen; Valencia, CA, USA). This study was approved by the Review Board of the Second Xiangya Hospital of the Central South University of China. For mutation analysis, the entire coding regions, including the flanking intronic sequences, of KRT1 (Refseq: NM_006121) and KRT9 (NM_000226) were amplified with polymerase chain reaction (PCR) (primer sequences will be provided upon request). Sequences of the PCR products were determined using the ABI 3100 Genetic Analyzer (ABI; Foster City, CA, USA) as previously described (Tan et al., 2012).

\section{RESULTS}

Mutational analysis identified a heterozygous $\mathrm{C}$ to $\mathrm{T}$ transition at nucleotide 487 that 
resulted in an arginine (R) to tryptophan (W) change at position 163 of KRT9 in all affected patients (Figure 1C). This p.R163W mutation has been reported previously in different races, and had been demonstrated as a hotspot mutation (Chiu et al., 2007; Codispoti et al., 2009; Xu et al., 2009). All of the affected familial members carried this mutation. We also identified a silent mutation $(1389 \mathrm{C} \rightarrow \mathrm{T}$ ) in exon 5 of $K R T 1$ in the proband (Figure 1C); this variant is not present in the 1000 genomes database or in the Single Nucleotide Polymorphism database (dbSNP).

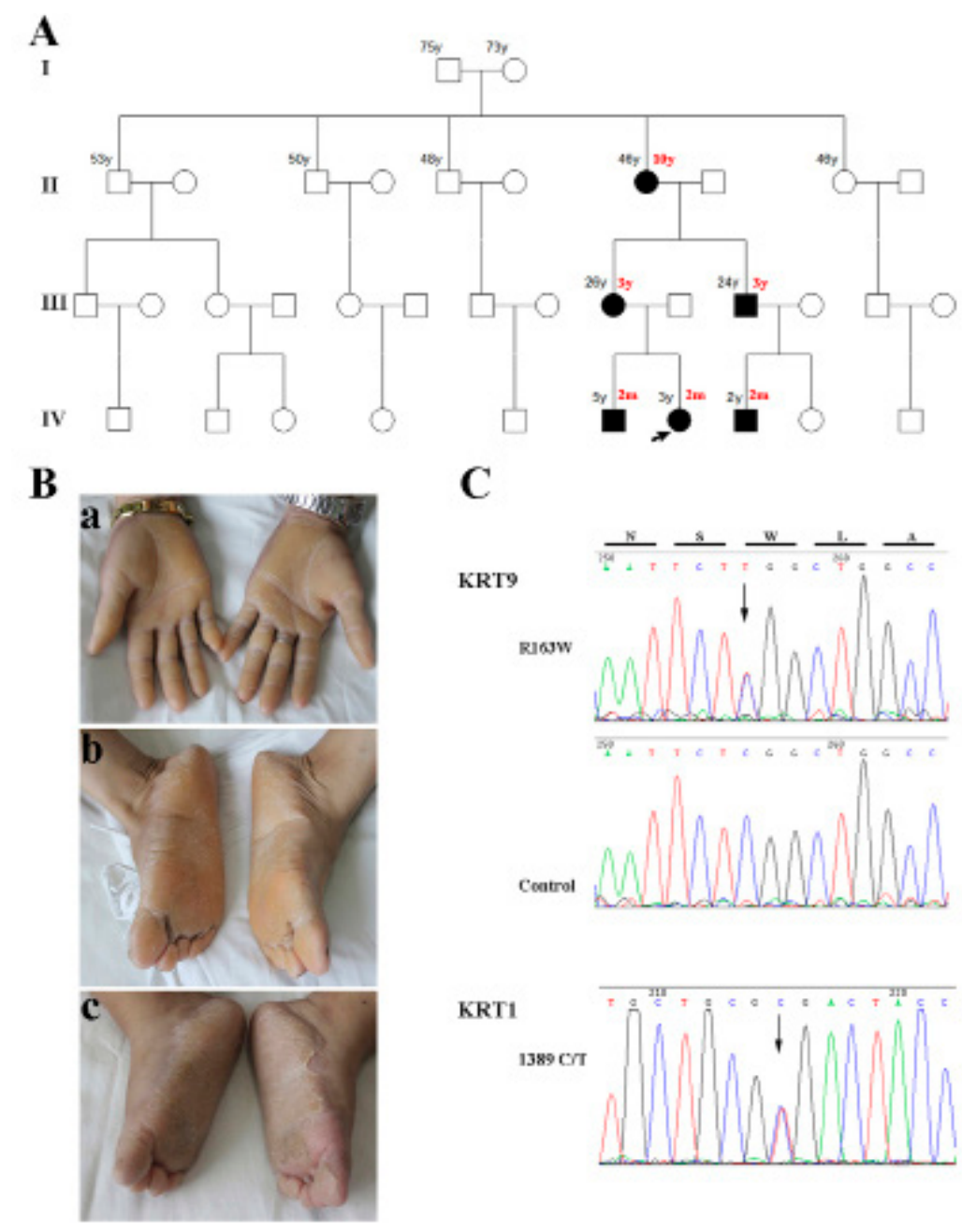

Figure 1. A. Pedigree of the family with epidermolytic palmoplantar keratoderma (EPPK). Arrow shows the proband, black symbols show the affected members. A decrease in the age of onset is observed in three consecutive generations (Generations II, III, and IV). Age of the individuals are indicated by the black color number in the upper left, age of onset of patients are indicated by the red color number. $y=$ year; $m=$ month. B. Clinical appearance of two of the affected members. a and b show the hands and feet of the proband's mother (III-9). c shows the feet of the proband (IV-7). Diffuse yellowish hyperkeratosis and scaling were present on both hands and feet, with an erythematous border. C. DNA sequencing result of $K R T 9$ and $K R T 1$. The upper arrow indicates a $\mathrm{C}$ to $\mathrm{T}$ substitution resulting in $\mathrm{R} 163 \mathrm{~W}$ in $K R T 9$; the lower arrow indicates a silent mutation (C to T). 


\section{DISCUSSION}

Of particular interest to our research group was the possible genetic anticipation in this EPPK family with the p.R163W mutation of KRT9. After critical examination of family members, we observed a decrease in the age of onset in three consecutive generations in the family of the proband. Age of disease onset varied from an adolescent of generation II to within the first two months after birth of generation IV (Figure 1A). The parents and siblings of the grandmother (II-7) of the proband were healthy. Given that EPPK is highly penetrant, the grandmother might carry a de novo p.R163W mutation in the KTR9 gene.

Genetic anticipation is a biological phenomenon in which the disease onset becomes progressively earlier or the disease is more severe in successive generations. Anticipation has been well recognized in a number of disorders including Huntington's disease (Ranen et al., 1995), familial chronic myeloproliferative disorders (Rumi et al., 2007), Lynch syndrome (Nilbert et al., 2009), familial hematologic malignancies (Tegg et al., 2011), and familial pancreatic cancer (McFaul et al., 2006). The underlying mechanisms for anticipation likely differ between different types of disorders. Expansion of trinucleotide repeat sequences, telomere shortening, and epigenetic factors are the three most common explanations for genetic anticipation (Nilbert et al., 2009).

Although ascertainment bias might be a consideration with respect to anticipation, we believe that in this family, there are several lines of evidence for the presence of anticipation. First, EPPK shows an early onset and highly penetrant manner, and most patients present EPPK from infancy. In the present case, EPPK is present from generation II, indicating that member II-7 of the family (the proband's grandmother) carries the de novo mutation. A similar observation was reported in a Japanese family with epidermolytic hereditary palmoplantar keratoderma (Sakabe et al., 2009). Second, EPPK exhibits an AD inheritance pattern, which is consistent with an anticipation model of a dominantly acting mutation (Tegg et al., 2011). Third, epigenetic factors, such as methylation and imprinting, might underlie the potential mechanism of anticipation. Interestingly, the p.R163W KRT9 mutation results in a CpG methylation site, and hence leads to chemical instability (Walsh and $\mathrm{Xu}, 2006$ ). Fourth, loss of a protective allele or another interacting gene was observed (Romero et al., 2008) as we found a variant of the $K R T 1$ gene in our proband.

In conclusion, we described an EPPK family with the p.R163W mutation of Keratin 9; we also identified a variant of the Keratin 1 gene $(1389 \mathrm{C} \rightarrow \mathrm{T})$ in the proband. After critical examination of family members, we observed a decrease in the age of onset in three consecutive generations in the family, indicating the presence of possible anticipation. Further studies are needed to elucidate the mechanisms of anticipation in EPPK.

\section{ACKNOWLEDGMENTS}

We thank the patient and her family for participating in this study. We thank the State Key Laboratory of Medical Genetics of China for technical assistance. Research supported by the National Natural Science Foundation of China (\#81101475).

\section{REFERENCES}

Chiu HC, Jee SH, Sheen YS, Chu CY, et al. (2007). Mutation of keratin 9 (R163W) in a family with epidermolytic 
palmoplantar keratoderma and knuckle pads. J. Dermatol. Sci. 45: 63-65.

Codispoti A, Colombo E, Zocchi L, Serra V, et al. (2009). Knuckle pads, in an epidermal palmoplantar keratoderma patient with Keratin 9 R163W transgrediens expression. Eur. J. Dermatol. 19: 114-118.

Hennies HC, Zehender D, Kunze J, Kuster W, et al. (1994). Keratin 9 gene mutational heterogeneity in patients with epidermolytic palmoplantar keratoderma. Hum. Genet. 93: 649-654.

Liu XP, Ling J, Xiong H, Shi XL, et al. (2009). Mutation L437P in the 2B domain of keratin 1 causes diffuse palmoplantar keratoderma in a Chinese pedigree. J. Eur. Acad. Dermatol. Venereol. 23: 1079-1082.

McFaul CD, Greenhalf W, Earl J, Howes N, et al. (2006). Anticipation in familial pancreatic cancer. Gut 55: 252-258.

Nilbert M, Timshel S, Bernstein I and Larsen K (2009). Role for genetic anticipation in Lynch syndrome. J. Clin. Oncol. 27: 360-364.

Ranen NG, Stine OC, Abbott MH, Sherr M, et al. (1995). Anticipation and instability of IT-15 (CAG)n repeats in parentoffspring pairs with Huntington disease. Am. J. Hum. Genet. 57: 593-602.

Reis A, Hennies HC, Langbein L, Digweed M, et al. (1994). Keratin 9 gene mutations in epidermolytic palmoplantar keratoderma (EPPK). Nat. Genet. 6: 174-179.

Romero P, Vogel M, Diaz JM, Romero MP, et al. (2008). Anticipation in familial lattice corneal dystrophy type I with R124C mutation in the TGFBI (BIGH3) gene. Mol. Vis. 14: 829-835.

Rumi E, Passamonti F, Della Porta MG, Elena C, et al. (2007). Familial chronic myeloproliferative disorders: clinical phenotype and evidence of disease anticipation. J. Clin. Oncol. 25: 5630-5635.

Sakabe J, Nakamura M and Tokura Y (2009). A missense mutation in exon 1 of the keratin 9 gene in a Japanese patient with "Vorner type" hereditary palmoplantar keratoderma. Eur. J. Dermatol. 19: 286-287.

Tan ZP, Huang C, Xu ZB, Yang JF, et al. (2012). Novel ZFPM2/FOG2 variants in patients with double outlet right ventricle. Clin. Genet. 82: 466-471.

Tegg EM, Thomson RJ, Stankovich JM, Banks A, et al. (2011). Anticipation in familial hematologic malignancies. Blood 117: 1308-1310

Terron-Kwiatkowski A, Terrinoni A, Didona B, Melino G, et al. (2004). Atypical epidermolytic palmoplantar keratoderma presentation associated with a mutation in the keratin 1 gene. Br. J. Dermatol. 150: 1096-1103.

Walsh CP and Xu GL (2006). Cytosine methylation and DNA repair. Curr. Top. Microbiol. Immunol. 301: 283-315.

Xu CM, Chen XL, Chen CY and Zhang XN (2009). Missense mutation of keratin 9 (c.487C $>$ T (p.R163W) in southern Chinese patients with epidermolytic palmoplantar keratoderma. Eur. J. Dermatol. 19: 265-266. 